\title{
AIAA 2001-0152
}

Acceleration of Convergence to a

Periodic Steady State in

Turbomachinery Flows

Matthew McMullen, Antony Jameson

and Juan J. Alonso

Stanford University, Stanford, CA 94305

39th AIAA Aerospace Sciences Meeting \& Exhibit

January 8-11, 2001/Reno, NV 
AIAA 2001-0152

\title{
Acceleration of Convergence to a Periodic Steady State in Turbomachinery Flows
}

\author{
Matthew McMullen, ${ }^{*}$ Antony Jameson ${ }^{\dagger}$ \\ and Juan J. Alonso \\ Stanford University, Stanford, CA 94305
}

\begin{abstract}
This paper presents a technique used to accelerate the convergence of unsteady calculations of time-periodic flows to a periodic steady state. The basis of the procedure is the use of the discrete Fourier transform in time, and is similar to the harmonic balance procedure that has been pursued by Hall et. al. The technique is amenable to parallel processing, and convergence acceleration techniques such as multi-grid and implicit residual averaging. The computational efficiency of this method is compared with dual time stepping algorithms. Sample calculations are provided, and a comparison between solutions with varying temporal resolution is presented. The results show that the computational efficiency of the harmonic balance technique is largely a function of the temporal resolution. Initial experiments confirm the promise of the harmonic balance method to achieve significant reductions in computational cost.
\end{abstract}

\section{Introduction}

The calculation of periodic unsteady flows in turbomachinery continues to present a severe challenge to Computational Fluid Dynamics (CFD). The unsteadiness stems mainly from the relative motion of the rotating blade fields, and has a fundamental period which depends on the rate of rotation and the number of blade passages. Currently, a popular approach to the computation of this problem is to introduce a fully implicit A-stable time discretization of the flow equations requiring the solution of a set of coupled nonlinear equations at each physical time step. These equations are typically solved with an explicit inner iteration by introducing a pseudo-time variable and marching to a steady state. Converged solutions attained at the end of the inner iterations represent a solution of the implicit equations at the end of the physical time step. ${ }^{1-4}$ A variety of techniques such as variable local pseudo-time steps, implicit residual averaging and multigrid are used to accelerate the convergence of the inner iterations. The efficiency of this dual time stepping method depends on the effectiveness of these acceleration techniques in restricting the number of inner iterations. If the physical time step can be increased in comparison to an explicit scheme by a factor larger than the number of inner iterations, the implicit scheme will be more efficient than the explicit scheme. In practice it has been found that for simple geometries and flows, on the order of 24-36 implicit time steps are sufficient to resolve the features

\footnotetext{
*Graduate Student, Student Member AIAA

$\dagger^{\top}$ T.V. Jones Professor of Engineering, AIAA Fellow

$¥$ Assistant Professor, AIAA Member

Copyright (C) 2001 by the authors. Published by the American Institute of Aeronautics and Astronautics, Inc. with permission.
}

of one period in the oscillation of the flow. Typically one restricts the number of inner iterations to the order of 30-80. At a minimum, capturing one period requires 1000 inner iterations. As the number of periods needed to obtain convergence increases the calculation can become prohibitively expensive. This is particularly true in multistage turbomachinery flows where convergence to a periodic steady state can be slow since it requires the physical propagation of disturbances back and forth between the front and the back of the turbomachinery component. The situation is exacerbated in complex turbomachinery geometries where 100 implicit time steps are often used to resolve a fundamental period of the blade passing frequency.

Another approach to the calculation of turbomachinery flows is to linearize the unsteady flow about a mean flow solution and solve for the unsteadiness in the flow using a frequency domain approach. ${ }^{5}$ Assuming that the disturbances are small compared to the mean flow values, the unsteady component of the solution can be expanded in a Fourier series and a decoupled equation is obtained for each of the fundamental modes in the expansion. This equation can be solved with small modifications to typical steadystate solvers. The computational cost is proportional to the cost of the steady-state solution multiplied by the number of distinct modes specified by a desired amount of temporal accuracy. Needless to say, the error in the linearization procedure becomes significant when the level of unsteadiness in the flow is substantial as it happens in most real-life viscous flow examples.

Hall et al, ${ }^{6}$ have proposed to apply the harmonic balance technique to the fully non-linear Navier-Stokes equations. For time-periodic flows, the method can 
be computationally more efficient than the dual-time stepping approach without the assumptions required by a linearized solution. Time periodicity is the fundamental property that permits this reduction in computational cost when compared with the dual-time stepping technique, which was designed for flows with arbitrary time histories. As with the linearized method the cost is proportional to the cost of the steady-state solution multiplied by the number of temporal modes required.

Time accurate solvers, such as those using the dualtime stepping approach, accurately capture the entire time history of the flow from initial guess to convergence to a periodic steady state. The decay rate of the transients associated with the initial guess can be significantly slowed down by the reflectivity of boundary conditions and geometric complexity. Harmonic balance techniques transform the unsteady equations in the physical domain into a steady problem in the frequency domain. The process of finding a steady solution to the transformed equations does not waste time resolving the initial transients and their decay since our interest lies on a time-periodic solution alone. It must be noted that the cost of the frequency domain solver is closely tied to the number of temporal modes needed to accurately resolve the flow phenomena. If the physical phenomena that one wants to resolve is such that only a few fundamental frequencies are present, frequency domain solvers can provide a significant computational advantage over more traditional techniques.

The purpose of this paper is to explore the potential advantages of frequency domain harmonic balance techniques to compute time-periodic unsteady flows of the sort that typically occur in multistage turbomachinery (compressors and turbines). Before attempting calculations of this complexity it seems well advised to validate the harmonic balance method for simpler periodic flows for which analytic solutions and experimental data are available. Furthermore, some of these simpler cases have been previously computed in our group using the dual-time stepping technique and can serve to provide direct cost comparisons between approaches. ${ }^{7,8}$ In this paper we focus on two test cases; one dimensional channel flow, and the vortex shedding behind a cylinder in the laminar regime.

\section{Governing Equations}

Viscous unsteady fluid flows in two dimensions can be described by the Navier Stokes equations presented in integral form below

$$
\int_{\Omega} \frac{\partial W}{\partial t} d V+\oint_{\partial \Omega} \vec{F} \cdot \vec{N} d s=0
$$

where

$$
\begin{aligned}
& W= {\left[\begin{array}{c}
\rho \\
\rho u \\
\rho v \\
\rho E
\end{array}\right] } \\
& \vec{F}_{1}=f=\left[\begin{array}{c}
\rho u \\
\rho u^{2}+p-\sigma_{x x} \\
\rho u v-\sigma_{x y} \\
\end{array}\right] \\
& \vec{F}_{2}=g=\left[\begin{array}{c}
\rho \sigma_{x x}-v \sigma_{x y}+q_{x} \\
\rho v \\
\rho u v-\sigma_{x y} \\
\rho v^{2}+p-\sigma_{y y} \\
\rho v H-u \sigma_{x y}-v \sigma_{y y}+q_{y}
\end{array}\right],
\end{aligned}
$$

and $\vec{N}$ is the outward pointing normal on the surface of the control volume. The variables $\rho, u, v$, and $E$ are density, Cartesian velocity components and specific total energy respectively. The flux terms also contain the thermodynamic pressure, $p$, the stress tensor, $\sigma$, and the heat flux vector obtained from Fourier's heat conduction law, $\vec{q}$. Closure is provided by the following equations:

$$
\begin{aligned}
p & =(\gamma-1) \rho\left[e-\frac{1}{2}\left(u^{2}+v^{2}\right)\right] \\
\sigma_{x x} & =2 \mu u_{x}-\frac{2}{3} \mu\left(u_{x}+v_{y}\right) \\
\sigma_{y y} & =2 \mu v_{y}-\frac{2}{3} \mu\left(u_{x}+v_{y}\right) \\
\sigma_{x y} & =\sigma_{y x}=\mu\left(u_{y}+v_{x}\right) \\
q_{x} & =\kappa \frac{\partial T}{\partial x}=-\frac{\gamma}{\gamma-1} \frac{\mu}{\operatorname{Pr}} \frac{\partial \frac{p}{\rho}}{\partial x} \\
q_{y} & =\kappa \frac{\partial T}{\partial y}=-\frac{\gamma}{\gamma-1} \frac{\mu}{\operatorname{Pr}} \frac{\partial \frac{p}{\rho}}{\partial y} .
\end{aligned}
$$

The physical domain of interest can be discretized such that a complete set of design variables resides at the center of each cell in the mesh. The volume of each cell is denoted by $V$. Applying Eq. 1 to each cell in the mesh, we can take the time derivative operator outside of the integral sign and the remaining integral can be approximated by the product of the cell volume and the current value of the flow solution at the cell center. The boundary integral is calculated by discrete integration of the fluxes around the control volume in a manner which is equivalent to central differencing. A modified JST scheme ${ }^{9,10}$ is implemented to add in third-order artificial dissipation for numerical stability. In the physical domain the integral expression in Eq. 1 can be written in semi-discrete form as

$$
V \frac{\partial W}{\partial t}+R(W)=0
$$

where

$$
R(W)=\sum_{j=1}^{n} \vec{F}_{j} \cdot \vec{S}_{j}
$$


Under the assumption that the solution is periodic over a given time period, we can transform the independent variables and residual terms into the frequency domain using a discrete Fourier transform. In our following discussion, the variables $\hat{W}_{k}$ and $\hat{R}_{k}$ represent the Fourier coefficients (for a given wavenumber $k$ ) of the Fourier transforms of $W$ and $R(W)$ respectively. Numerically this transformation is accomplished using the Fast Fourier Transform (FFT) in order to minimize the cost of computation. The computational cost of this transform is proportional to $N \log N$, where $N$ is the number of time intervals used to describe the signal. For real solutions the Fourier coefficients for the negative wavenumbers are simply the complex conjugates of the coefficients for the positive wavenumbers. In taking advantage of this property we can eliminate half of the wavenumbers from the computation. Once we have obtained the Fourier coefficients for the expansions of $W$ and $R(W)$, we can recover these quantities using the inverse discrete Fourier transform as follows:

$$
\begin{aligned}
W & =\sum_{k=-\frac{N}{2}}^{\frac{N}{2}-1} \hat{W}_{k} e^{i k t} \\
R(W) & =\sum_{k=-\frac{N}{2}}^{\frac{N}{2}-1} \hat{R}_{k} e^{i k t},
\end{aligned}
$$

where

$$
i=\sqrt{-1} \text {. }
$$

If we apply the discrete Fourier transform to the semidiscrete form of the governing equation in Eq. 4, and we move the time derivative of the state variable inside the series summation, orthogonality of the Fourier series ensures that the individual contributions from each wavenumber is equal to zero

$$
i k V \hat{W}_{k}+\hat{R}_{k}=0 .
$$

It follows that a periodic steady-state equation can be written for each independent wavenumber. Direct solution of this equation is appropriate for all wavenumbers except for that associated with the mean value $(k=0)$. Instead of directly solving Eqs. 7 we add in a pseudo-time derivative and numerically integrate the resulting equations.

$$
V \frac{d \hat{W}_{k}}{d \tau}+i k V \hat{W}_{k}+\hat{R}_{k}=0
$$

Note, however, that solution of the physical problem will require iteration between physical and Fourier spaces, since, due to the nonlinearity of the residual operator, $R(W), \hat{R}_{k}$ cannot be computed directly from $\hat{W}_{k} \cdot \hat{R}_{k}$ can only be computed by physical evaluation of the residual at a number of time locations within one periodic cycle and the subsequent transformation to Fourier space using the discrete Fourier transform.
Figure 1 provides a flow chart of the data and transforms used to advance the solution through one iteration in pseudo time. At the beginning of this iteration we know $\hat{W}$ at every grid point for all wavenumbers. This initial guess can simply result from the discrete Fourier transform of a constant uniform flow. Using an inverse FFT we can transform $\hat{W}$ back to the state vector in physical space. The simulation now holds $W$ at every grid point and instance in time. At each of the points in time being resolved in the simulation, we compute the steady-state residual $R$, and, using an FFT we transform it back to the frequency domain to obtain $\hat{R}$. We calculate the overall residual by adding $\hat{R}$ to the source term $i k V \hat{W}$. This overall residual, specified in the frequency domain, is used to compute the new approximate $\hat{W}$, and the process returns to the beginning of the cycle.

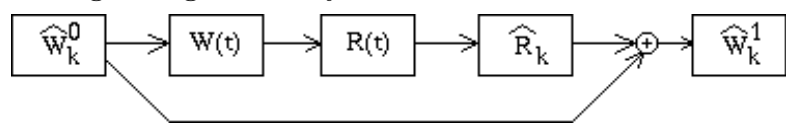

Fig. 1 Process Flowchart

\section{Farfield Boundary Conditions}

During the process of development of the harmonic balance technique, we found that the implementation of proper far-field boundary conditions was necessary to obtain convergence for the unsteady components of the solution. For this reason, this section describes in some detail the far-field boundary conditions that have been implemented into our code.

At the boundary of the domain we assume that the viscous terms in the stress tensor are negligible in comparison to the convective fluxes. We also assume that the unsteady perturbations in the flow are small in comparison to the average values. Given these assumptions we apply the method of Riemann invariants on a two-dimensional boundary to determine the mean flow solution. The unsteady perturbations are calculated using Giles'11 boundary conditions applied to the solution in the frequency domain.

The unsteady boundary conditions are derived by substituting the linearized state vector into the Euler equations written in the computational space $(\xi, \eta)$ :

$$
\begin{array}{r}
W=\bar{W}+\dot{W} \\
V \frac{\partial W}{\partial t}+\frac{\partial f_{1}}{\partial \xi}+\frac{\partial g_{1}}{\partial \eta}=0
\end{array}
$$

where

$$
\begin{aligned}
f_{1} & =f \frac{\partial y}{\partial \eta}-g \frac{\partial x}{\partial \eta} \\
g_{1} & =-f \frac{\partial y}{\partial \xi}+g \frac{\partial x}{\partial \xi}
\end{aligned}
$$

Due to the assumption of linearity the mean flow terms drop out of the resulting equation. Higher order perturbations are eliminated leaving only first order terms 
in the flux vectors. The Jacobian matrices are calculated as the derivatives of these flux vectors with respect to the perturbed variable, leaving only mean flow variables in the definition of the Jacobians:

$$
V \frac{\partial \dot{W}}{\partial t}+\bar{A} \frac{\partial \dot{W}}{\partial \xi}+\bar{B} \frac{\partial \hat{W}^{\prime}}{\partial \eta}=0
$$

where

$$
\begin{aligned}
\bar{A} & =\frac{\partial \dot{f}}{\partial \dot{W}} \frac{\partial y}{\partial \eta}-\frac{\partial \dot{g}}{\partial \dot{W}} \frac{\partial x}{\partial \eta} \\
\bar{B} & =-\frac{\partial \dot{f}}{\partial \dot{W}} \frac{\partial y}{\partial \xi}+\frac{\partial \dot{g}}{\partial \dot{W}} \frac{\partial x}{\partial \xi}
\end{aligned}
$$

Since the Jacobians are independent of the perturbed variables they act as simple linear operators on these vectors. Consequently we can transform the perturbed variables to the frequency domain using a straightforward Fourier transform. Taking advantage of the system's linearity and the orthogonality of the Fourier series, we can eliminate the summation operator and the complex exponential term

$$
\begin{aligned}
\dot{W} & =\sum_{k=-\frac{N}{2}}^{\frac{N}{2}-1} \tilde{W}_{k} e^{i k t} \\
i k V \tilde{W}_{k} & +\bar{A} \frac{\partial \tilde{W}_{k}}{\partial \xi}+\bar{B} \frac{\partial \tilde{W}_{k}}{\partial \eta}=0 .
\end{aligned}
$$

We assume that the derivatives with respect to $\eta$ are constant in the direction normal to the boundary. Working in eigenspace, for a two dimensional flow, the equations decouple into four independent ordinary differential equations. A solution can be obtained by integrating between two arbitrary points

$$
Y_{j}(\xi)=-\frac{C_{j}(0)}{i k V}+\left[Y_{j}(0)+\frac{C_{j}(0)}{i k V}\right] e^{-\frac{i k V}{\Lambda_{j}}},
$$

where

$$
\begin{aligned}
\bar{A} & =X \Lambda X^{-1} \\
Y & =X^{-1} \tilde{W}_{k} \\
C & =X^{-1} \bar{B} \frac{\partial \tilde{W}_{k}}{\partial \eta} .
\end{aligned}
$$

In practice, the final interior point to the domain serves as a basis to evaluate all of the terms in the equation. The characteristic solutions associated with the outgoing waves are extrapolated to the ghost cell. Any eigenvectors associated with incoming characteristics are specified at the ghost cell. The new state vector at the ghost cell is then reconstructed as a linear combination of the eigenvectors.

For the unsteady one-dimensional duct problem, there is no variation in the flow solution with respect to $\eta$. Since the $C_{j}$ term is a function of $\frac{\partial \tilde{W}}{\partial \eta}$, it is consequently zero. At the exit of the duct we can write an explicit equation to recombine the eigenvectors at the ghost cell; note that the subscripts $i e$ and $i l$ denote the ghost cell and first interior cell respectively. The superscripts $n$ and $n+1$ denote the value of the eigenvector before and after application of the boundary condition.

$$
\begin{aligned}
Y_{i e}^{n+1} & =\Lambda_{+} Y_{i l}^{n}+\Lambda_{-} Y_{i e}^{n} \\
\Lambda_{+} & =\left[\begin{array}{ccc}
e^{\frac{-i k \Delta x}{\bar{u}}} & 0 & 0 \\
0 & e^{\frac{-i k \Delta x}{u+\bar{c}}} & 0 \\
0 & 0 & 0
\end{array}\right] \\
\Lambda_{-} & =\left[\begin{array}{lll}
0 & 0 & 0 \\
0 & 0 & 0 \\
0 & 0 & 1
\end{array}\right] .
\end{aligned}
$$

The characteristic value at the ghost cell is computed as a combination of the exit pressure perturbation specified by the boundary conditions and the current values of the state vector at the ghost cell.

$$
Y_{i e}^{n}=X^{-1}\left[\begin{array}{c}
\tilde{\rho}_{i e}^{n} \\
\tilde{\rho} u_{i e}^{n} \\
\frac{p_{e}}{\gamma-1}-\frac{1}{2} \bar{u}^{2} \tilde{\rho}_{i e}^{n}+\bar{u} \tilde{\rho} u_{i e}^{n}
\end{array}\right] .
$$

If the right hand vector contained in the above equation is split into the following sum

$$
\begin{aligned}
Y_{i e}^{n} & =X^{-1} B \tilde{W}_{i e}^{n}+X^{-1} C \\
B & =\left[\begin{array}{ccc}
1 & 0 & 0 \\
0 & 1 & 0 \\
-\frac{1}{2} \bar{u}^{2} & \bar{u} & 0
\end{array}\right] \\
C & =\left[\begin{array}{c}
0 \\
0 \\
\frac{\dot{p}_{e}}{\gamma-1}
\end{array}\right]
\end{aligned}
$$

then we can write an implicit equation for the boundary conditions. This form is advantageous in the fact that the ghost cell value is a function only of the interior cell values and boundary condition constants:

$$
\begin{aligned}
\tilde{W}_{i e}^{n+1} & =\left(I-A_{-} B\right)^{-1}\left(A_{+} \tilde{W}_{i l}^{n}+A_{-} C\right) \\
A_{+} & =X \Lambda_{+} X^{-1} \\
A_{-} & =X \Lambda_{-} X^{-1} .
\end{aligned}
$$

For the channel flow problem, the improved convergence rates provided by the implicit form justify the additional computational cost required in solving a linear system of equations. The equation for the inlet is similar except that there is only one outgoing characteristic which has speed $\bar{u}-\bar{c}$. The characteristic value at the inlet ghost cell (denoted 1 ) is formed to hold stagnation pressure constant:

$$
Y_{1}^{n}=X^{-1}\left[\begin{array}{c}
\tilde{\rho}_{1}^{n} \\
\frac{1}{2} \bar{u} \tilde{\rho}_{1}^{n}+\underset{\substack{\gamma-2 \\
\gamma E_{1}^{n}}}{\left(\frac{\gamma-1}{\rho} E_{1}^{n}\right.} \\
\bar{u}
\end{array}\right] .
$$


Forming an implicit equation for the inlet boundary condition is similar to the steps used for the exit condition. For the cylinder model the radiation boundary condition assumes the incoming characteristic is zero. The physical value at the ghost cell is reconstructed using extrapolations based only on outgoing characteristics. Since the incoming characteristic is ignored no implicit formulation is necessary.

\section{Solution of the Field Equations Stability}

In this section we examine the effect of the harmonic balance technique on the stability of the pseudo-time Eq. 8. For simplicity we assume a linearization using only one space dimension:

$$
\frac{d \hat{W}_{k}}{d \tau}+i k \hat{W}_{k}+A \frac{\partial \hat{W}_{k}}{\partial x}=0
$$

The $A$ matrix is the Jacobian of the flow equations. This equation can be further simplified using a von Neumann test whereby we expand the solution using a single spatial Fourier mode:

$$
\frac{d \check{W}}{d \tau}+i k \check{W}+\frac{M}{\Delta x} \check{W}=0 .
$$

Note that $\check{W}$ is the new solution variable, and $\mathrm{M}$ is the spectral matrix corresponding to the spatial discretization. For example with central differences

$$
M=A i \sin (\omega \Delta x),
$$

where $\omega$ is the spatial frequency. The maximum eigenvalue of $M$ is denoted as $\bar{\lambda}$. For stability in steadystate calculations, this eigenvalue divided by the mesh spacing, $\frac{\bar{\lambda}}{\Delta x}$, should lie in the stability region of the time stepping scheme. If we split this eigenvalue into real $\bar{\lambda}_{r}$ and imaginary $\bar{\lambda}_{i}$ parts, we arrive at a simplified equation containing the additional terms introduced by the harmonic balance technique:

$$
\frac{d \dot{W}}{d \tau}=-\left[\frac{\bar{\lambda}_{r}}{\Delta x}+\left(\frac{\bar{\lambda}_{i}}{\Delta x}+k\right) i\right] \dot{W} .
$$

Note that the new variable $\dot{W}$ is the characteristic variable formed by the multiplication of the eigenvectors of $M$ with $\check{W}_{k}$. Two observations are immediately clear. Harmonic balance does not affect the stability of the mean flow solution. Second, harmonic balance affects only the imaginary part of the eigenvalue associated with the time averaged solution. This effect is small for the lower temporal frequencies but cannot be ignored, as the higher frequencies will eventually produce a restriction in the stability limit. This stability restriction is quite similar to the one imposed by the original dual-time stepping method, ${ }^{1}$ only it shifts the Fourier symbol of the residual along the imaginary axis direction. Reduced CFL numbers may be necessary to ensure stability of the solution for high wavenumbers. Alternatively, in a manner similar to that proposed by Melson et al. ${ }^{12}$ for the dual-time stepping method, the $i k \breve{W}$ term can be treated point-implicitly in the Runge-Kutta iteration to ensure stability for the usual CFL limit of the time stepping scheme.

\section{Accuracy}

For this subsection the advection equation in onedimension is used as a model to develop estimates for the temporal and spatial accuracy of the numerical scheme. The boundary condition specifies the solution at the inflow as a periodic function in time:

$$
\begin{aligned}
\frac{\partial u}{\partial t}+c \frac{\partial u}{\partial x} & =0 \\
u(0) & =f(t) .
\end{aligned}
$$

Due to the linearity of the model equation, an exact solution (in the frequency domain) can be derived as a function of each wavenumber

$$
\hat{u}_{k}\left(x_{f}\right)=\hat{u}_{k}\left(x_{i}\right) e^{\frac{-i k\left(x_{f}-x_{i}\right)}{c}} .
$$

The solution to the discretized equation will be denoted as $w$. Assuming a central difference operator, the discrete equation in the frequency domain is provided as

$$
\frac{c}{2 \Delta x} \hat{w}_{k_{j+1}}+i k \hat{w}_{k_{j}}-\frac{c}{2 \Delta x} \hat{w}_{k_{j-1}}=0 .
$$

Assuming a semi-infinite domain, we can use a shift operator to find an analytic expression for the solution of the difference equation:

$$
\begin{aligned}
\hat{w}_{k_{j+n}} & =\hat{w}_{k_{j}} \sigma^{n} \\
\sigma & =-i \gamma \pm \sqrt{1-(\gamma)^{2}} \\
\gamma & =\frac{k \Delta x}{c} .
\end{aligned}
$$

The negative root of the above quadratic expression is spurious. Using a Taylor series expansion we can rewrite $\sigma$ as the combination of an exponential term and additional error components:

$$
\begin{aligned}
\sigma & =e^{-i \gamma}-\alpha e^{i \theta}+h o t \\
\alpha & =\sqrt{\left(\frac{\gamma^{3}}{6}\right)^{2}+\left(\frac{\gamma^{4}}{6}\right)^{2}} \approx \frac{|\gamma|^{3}}{6} \\
\theta & =\tan ^{-1}\left(\frac{1}{\gamma}\right)
\end{aligned}
$$

We denote the Fourier coefficient of the boundary condition as $\hat{f}_{k}$. Subtracting the analytic and discrete solutions provides an estimate for error at an arbitrary point in space.

$$
\hat{E}_{k}(x)=\hat{w}_{k}(x)-\hat{u}_{k}(x)=\left(\sigma^{n}-e^{\frac{-i k x}{c}}\right) \hat{f}_{k} .
$$




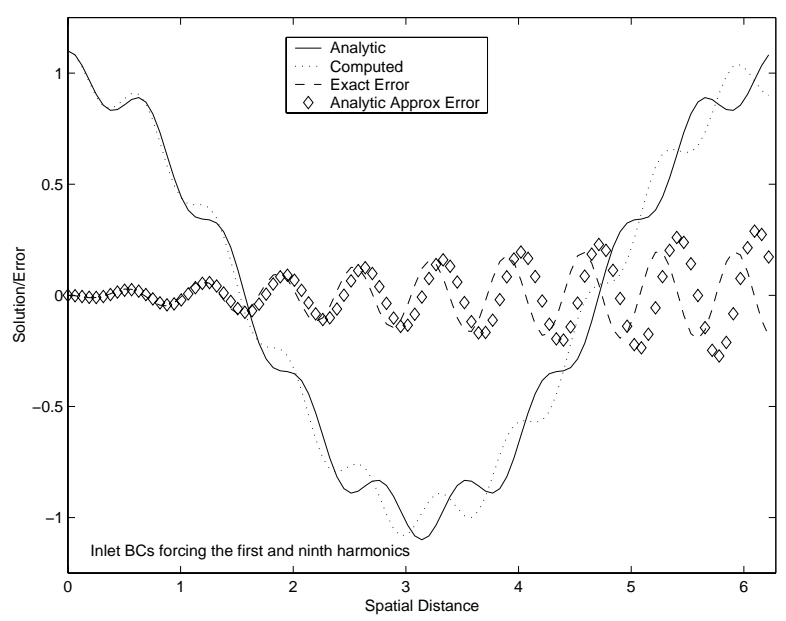

Fig. 2 Solution and Error for the Advection Equation

Note that the $\sigma$ term contains a binomial expansion. The binomial expansion contains terms that are products of factorials based on $n$ and various powers of $\alpha$. As $n$ becomes large the factorial coefficients dominate and more terms in the Taylor series expansion are needed to properly evaluate the error. For small $n$ (and small $x$ ) we retain only the first term and provide a useful estimate for error

$$
\hat{E}_{k}(x)=\left[-\frac{x}{6}\left|\frac{k}{c}\right|^{3} \Delta x^{2} e^{i\left(\frac{-k x}{c}+\gamma+\arctan \frac{1}{\gamma}\right)}\right] \hat{f}_{k} .
$$

Predictably, the magnitude of the error is a function of $\Delta x^{2}$. The phase shift is represented by the exponential term and grows linearly with $x$. The above approximation shows no error as a function of time. This assumes that all of the temporal modes excited at the boundary are being resolved. If this is not the case, then the unresolved modes will alias error back into the solution.

A numerical experiment was performed to check the accuracy of the above approximation. In Figure 2 the analytic solution to the advection equation is plotted as a solid line, the discrete solution is plotted as dots. The exact difference between these two solutions is plotted as a dashed line. The results produced by Eq. 30 are plotted on the graph as diamonds.

Two different frequencies were excited at the inflow boundary and the results were plotted for the entirety of the larger wavelength. Note that the above equation accurately predicts error for small $x$. For larger $x$, the real error growth slows down and no longer follows the linear relationship predicted by the above expression. This error is largely a function of the phase shift in the wave form caused by dispersive terms. The overall shape of the waveform is in general preserved due to the absence of dissipation in the spatial differencing scheme.

\section{Convergence Acceleration}

The transformed equations represent the solution of a steady system of equations in the frequency domain. This facilitates applying established convergence acceleration techniques to improve computational efficiency. A V-cycle full multigrid scheme with variable local pseudo-time stepping and implicit residual averaging was implemented. The solution was advanced in pseudo time using a modified multi-step RK scheme. Except for the residual averaging operations, the explicit nature of the time advancement scheme facilitated the parallelization of this code. The current solver utilizes multiple blocks distributed on different processors. A dual halo scheme serves to locally cache the state vector from neighboring grids. MPI libraries implement the actual communication between processors. The amount of inter-block communication scales linearly with the number of wavenumbers the user specifies a priori.

\section{Results}

\section{Unsteady Channel Flow}

The first test used in the verification of this method has an analytic solution. The test problem is the simulation of incompressible inviscid flow through a one dimensional channel. The physical analogue to this problem is pictured in Figure 4.

Assuming small perturbations, Merkle and Athavale $^{13}$ proposed a solution for the flow field. Ignoring the time transient terms associated with the initial guess, the periodic steady state solution is:

$$
\begin{aligned}
\dot{u}(t) & =-\frac{\dot{p}_{e}}{\rho \bar{u}} \frac{1}{1+\Omega^{2}}[\sin (w t)-\Omega \cos (w t)] \\
\Omega & =\frac{w L}{\bar{u}} \\
\dot{p}(x, t) & =\dot{p}_{e} \sin (w t) \\
& +(x-L) \frac{\dot{p}_{e}}{L} \frac{\Omega}{1+\Omega^{2}}[\Omega \sin (w t)+\cos (w t)] .
\end{aligned}
$$

Conservation of mass dictates that velocity is constant along the length of the duct. However, pressure varies linearly along the duct length. Both quantities vary sinusoidally with time.

A numerical experiment was performed to compare the analytic results with the full non-linear harmonic balance code. The code was run using the following physical parameters:

$$
\begin{aligned}
\text { Pressure Pertubation } \frac{p_{e}}{\bar{p}} & =1 e-4 \\
\text { Mach Number } \bar{M} & =0.1 \\
\text { Reduced Frequency } \Omega & =1 .
\end{aligned}
$$

Numerical solution fields plotted in space and time are provided in Figs. 5 and 6. Cross sections of the normalized error at different instances in time are provided 


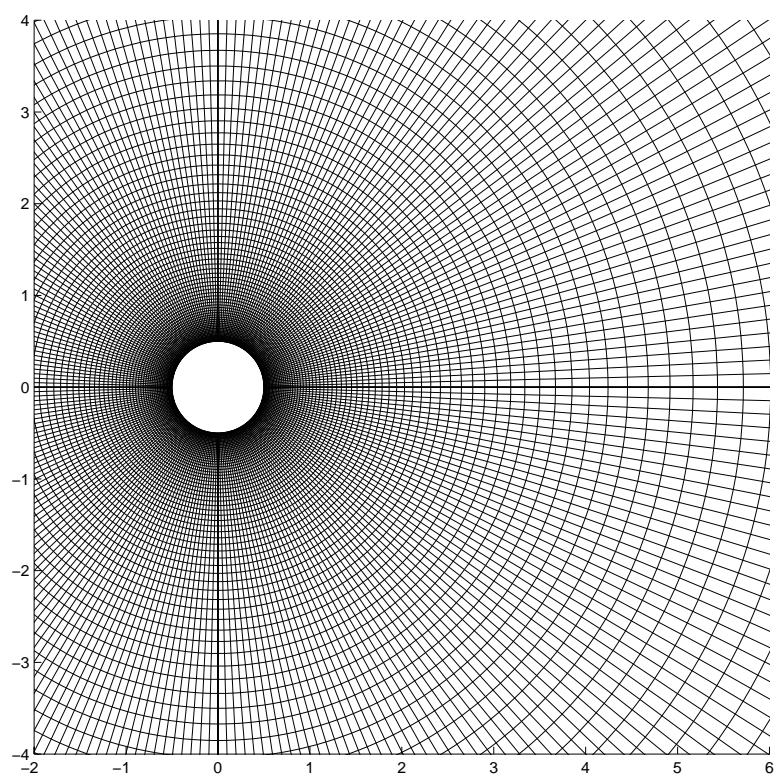

Fig. 3 Computational Grid for Cylinder Flow

in Figs. 7 and 8. These plots show that the maximum error for both pressure and velocity typically occurs at the inlet of the channel. To non-dimensionalize these results we normalized the error by the exit pressure perturbation, or the maximum velocity perturbation. Plotting these normalized errors at different instances in time shows that the maximum error never exceeds $2 \%$. Convergence history results are provided in Figure 9. Due to the linear nature of the solutions with respect to $x$, a first order scalar dissipation scheme was used. The model used a three level multigrid V-cycle to accelerate convergence. Machine zero residuals were obtained in 400 cycles. Selection of the dissipation scheme has obvious impacts on convergence, with higher order dissipation schemes (used in the cylinder flow) contributing to slower convergence rates.

\section{Cylinder Flow}

The solution in the channel flow test case contains unsteady perturbations small enough to be modeled using linear theory. We choose our second test case as circular cylinder flow at a Reynolds number of 180 . The non-linear capabilities of the harmonic balance solver are tested by the unsteady perturbations associated with the Karman vortex street. Figure 3 provides a plot of the computational mesh directly surrounding the cylinder.

The grid dimensions are 256 by 128 points in the circumferential and radial directions respectively. The mesh boundary is 200 chords from the center of the cylinder. An exponential stretching function is used in the radial direction with the smallest grid spacing of $3.54 e-03$ chords occurring at the wall. At the top of the cylinder roughly 15 grid points captured the boundary layer. Table 1 provides a list of time averaged results from both physical experiment (first three) and numerical computation. A numerical ex-

\begin{tabular}{|l|l|l|l|}
\hline Experiment & $-C_{p b}$ & $C_{d}$ & $S_{t}$ \\
\hline \hline Williamson and Roshko $^{14}$ & 0.83 & & \\
\hline Roshko $^{15}$ & & & 0.185 \\
\hline Wieselsberger $^{16}$ & & 1.3 & \\
\hline Henderson $^{17}$ & 0.83 & 1.34 & \\
\hline
\end{tabular}

\section{Table 1 Time Averaged Experimental Data}

periment was conducted to determine the effect of the temporal resolution on the time averaged data. Using the same grid and boundary conditions we varied the number of temporal modes used in each calculation. Table 2 shows the results of these experiments for base pressure coefficient and coefficient of drag. The number provided in the temporal modes column does not include the time averaged mode $(k=0)$. In addition, there is no Stroudhal column in the following table because this parameter was specified a priori. The Stroudhal value used for this numerical experiment is 0.185 .

\begin{tabular}{|l|l|l|}
\hline Temporal Modes & $-C_{p b}$ & $C_{d}$ \\
\hline \hline 1 & 0.832 & 1.257 \\
\hline 3 & 0.895 & 1.306 \\
\hline 5 & 0.903 & 1.311 \\
\hline 7 & 0.903 & 1.311 \\
\hline
\end{tabular}

Table 2 Time Averaged Data versus Temporal Modes

Convergence data for the cylinder test case is problematic. Given an initial uniform flow field, the solution converged to a steady symmetrically separated flow; similar to those achieved at lower Reynolds numbers. The flow field was then perturbed with user intervention. The solution then converged to the classic periodic shedding similar to physical flows at that Reynolds number. Given an initial solution that already contained vortex shedding, the single harmonic case used 600 multigrid cycles, with 5 levels, to lower the residual by two orders of magnitude. Convergence rates slowed as the number of temporal modes was increased.

Contour plots of the solution field are provided in Figures 10 through 17 . The left hand side figures represent the solution at an initial time. As one moves to the right, the flow evolves over the full period of oscillation. Two sets of contour plots are provided in this group of figures. The sets show the degradation in the solution features from the lowest temporal resolution case containing one harmonic to the highest temporal resolution case containing seven harmonics. Obviously, increasing the number of wavenumbers in the solution increases the detail and resolution of the features in the unsteady wake. 


\section{Conclusions}

An unsteady solver for the fully non-linear viscous Navier-Stokes equations has been developed. Harmonic balance techniques are used to transform the unsteady equations in the physical domain into a steady system in the frequency domain. The solver takes advantage of parallelizable convergence acceleration techniques, such as multigrid, to find an efficient solution to the transformed equations. To verify the code, numerical experiments were performed on an unsteady channel and a cylinder shedding periodic vortices. The results showed very good agreement with the analytic and experimental solutions. Further numerical experiments showed that a limited number of temporal modes could accurately resolve time averaged flow field quantities.

The results confirm the promise of the harmonic balance method to achieve significant reductions in computational cost, particularly in cases where only a small number of modes are sufficient to represent the periodic flow. Work is ongoing to extend this code to real applications in turbomachinery. At the very least, we believe that it could be very beneficial to use the harmonic balance method to provide an initial solution for fully time accurate unsteady simulations. This should eliminate the need to integrate the solution over a large number of periods before reaching a periodic steady state.

\section{Acknowledgements}

This work was sponsored by the Department of Energy's Accelerated Strategic Computing Initiative (ASCI). Special thanks to Kenneth Hall for his technical presentation and personal communications on this subject.

\section{References}

${ }^{1}$ A. Jameson. Time Dependent Calculations Using Multigrid, with Applications to Unsteady Flows Past Airfoils and Wings. Technical Report 91-1596, AIAA 10th Computaional Fluid Dynamics Conference, June 1991.

${ }^{2}$ A. Arnone, M. Liou, and L.A. Povinelli. Integration of Navier-Stokes Equations using Dual Time Stepping and a Multigrid Method. AIAA Journal, 33(6):985-990, June 1995.

${ }^{3}$ R.L. Davis, T. Shang, J. Buteau, and R. Ni. Prediction of 3-d Unsteady Flow in Multi-Stage Turbomachinery using an Implicit Dual Time-Step Approach. Technical Report 96-2565, AIAA, ASME, SAE, and ASEE 32nd Joint Propulsion Conference and Exhibit, Lake Buena Vista, FL, July 1996.

${ }^{4}$ J. Yao, A. Jameson, J.J. Alonso, and F. Liu. Development and Validation of a Massively Parallel Flow Solver for Turbomachinery Flows. Technical Report 00-0882, AIAA 38th Aerospace Sciences Meeting and Exhibit, January 2000.

${ }^{5}$ K.C. Hall and W.S. Clark. Linearized Euler Predictions of Unsteady Aerodynamic Loads in Cascades. AIAA Journal, 31(3):540-550, March 1993.

${ }^{6}$ K.C. Hall. Seminar on Harmonic Balance Techniques, January 2000. Presented to the Aerospace Computing Lab, Stanford University.

${ }^{7}$ J. J. Alonso, L. Martinelli, and A. Jameson. Multigrid unsteady Navier-Stokes calculations with aeroelastic applications.
AIAA paper 95-0048, AIAA 33rd Aerospace Sciences Meeting, Reno, NV, January 1995.

${ }^{8}$ A. Belov, L. Martinelli, and A. Jameson. A new implicit algorithm with multigrid for unsteady incompressible flow calculations. AIAA paper 95-0049, AIAA 33rd Aerospace Sciences Meeting, Reno, NV, January 1995.

${ }^{9}$ A. Jameson. Analysis and Design of Numerical Schemes for Gas dynamics I Artificial Diffusion, Upwind Biasing, Limiters and their Efect on Accuracy and Multigrid Convergence. International Journal of Computational Fluid Dynamics, 4:171-218, 1995.

${ }^{10}$ R.C. Swanson and E. Turkel. On Central-Difference and Upwind Schemes. Journal of Computational Physics, 101:297306, 1992.

${ }^{11}$ M.B. Giles. Nonreflecting Boundary Conditions for Euler Equation Calculations. AIAA Journal, 28(12):2050-2058, 1990.

${ }^{12}$ N. D. Melson, M. D. Sanetrik, and H. L. Atkins. Timeaccurate Navier-Stokes calculations with multigrid acceleration. In Proceedings of the Sixth Copper Mountain Conference on Multigrid Methods, Copper Mountain, April 1993.

${ }^{13}$ C.L. Merkle and M. Athavale. Time-Accurate Unsteady Incompressible Flow Algorithms Based on Artficial Compressbility. AIAA paper 87-1137, AIAA 8th Computational Fluid Dynamics Conference, 1987.

${ }^{14}$ C.H.K. Williamson and A. Roshko. Measurements of Base Pressure in the Wake of a Cylinder at Low Reynolds Numbers. Z. Flugwiss. Weltraumforsch, 1990.

${ }^{15}$ Anatol Roshko. On the Development of Turbulent Wakes from Vortex Streets. NACA Technical Report 1191, NACA, January 1954.

${ }^{16}$ C. Wieselsberger. New Data on the Laws of Fluid Resistance. Technical report, NACA, 1922.

${ }^{17}$ R.D. Henderson. Details of the Drag Curve Near the Onset of Vortex Shedding. Physics of Fluids, 1995. 


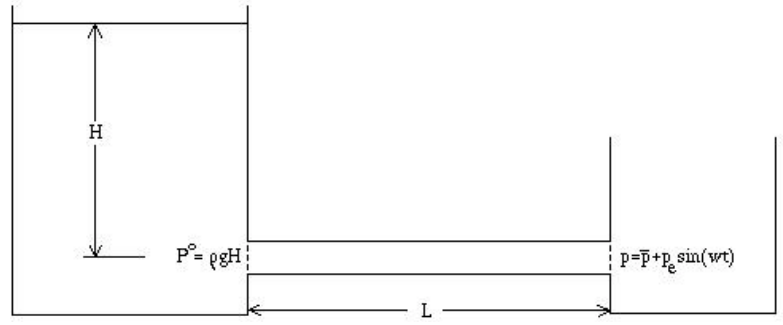

Fig. 4 One Dimensional Channel Flow

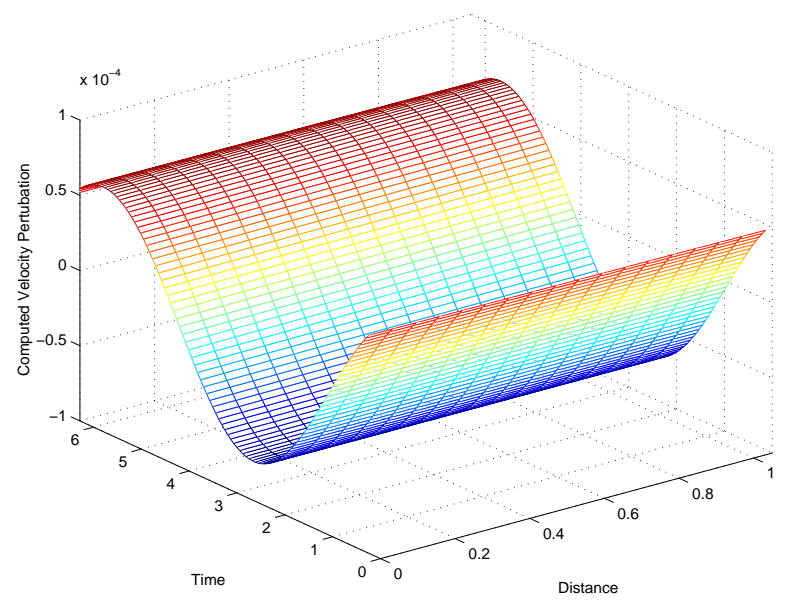

Fig. 5 Velocity in Space/Time for Channel Flow



Fig. 6 Pressure in Space/Time for Channel Flow

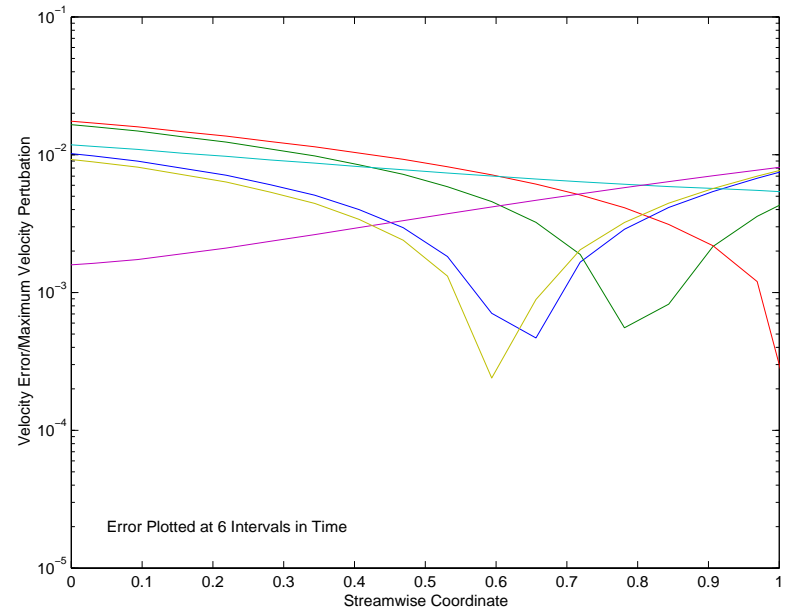

Fig. 7 Normalized Velocity Error for Channel Flow



Fig. 8 Normalized Pressure Error for Channel Flow

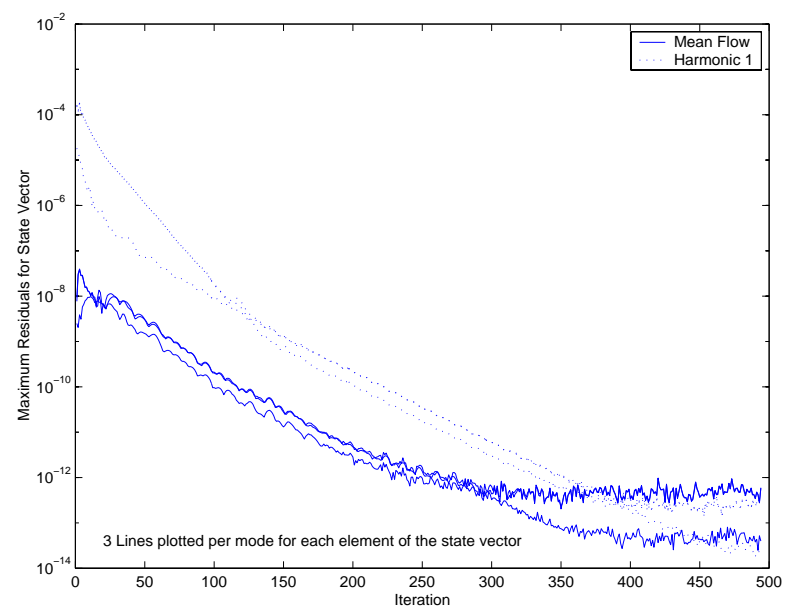

Fig. 9 Residual History for Channel Flow 

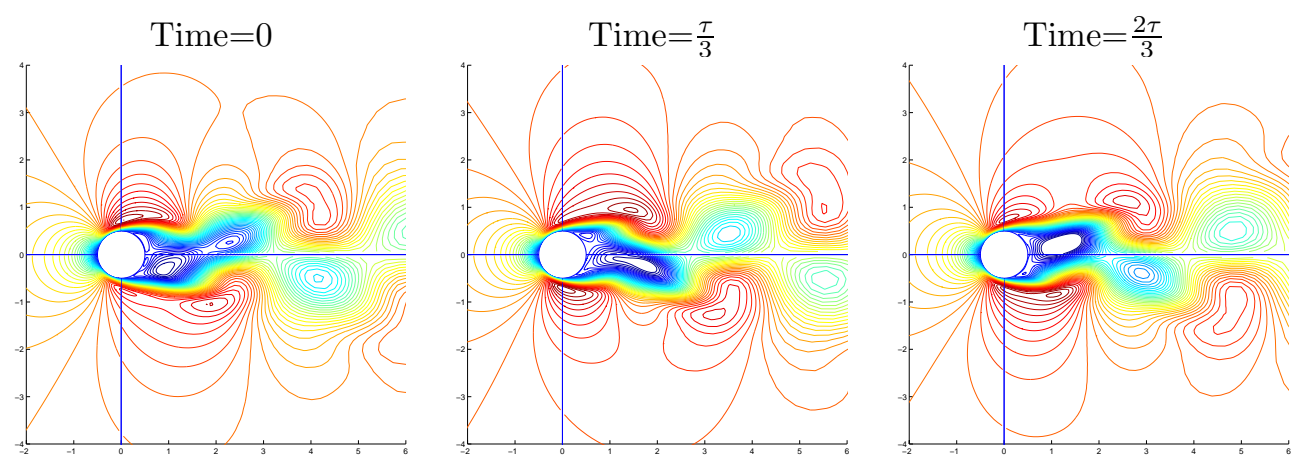

Fig. 10 Contours of the X Component of Velocity - 1 Harmonic
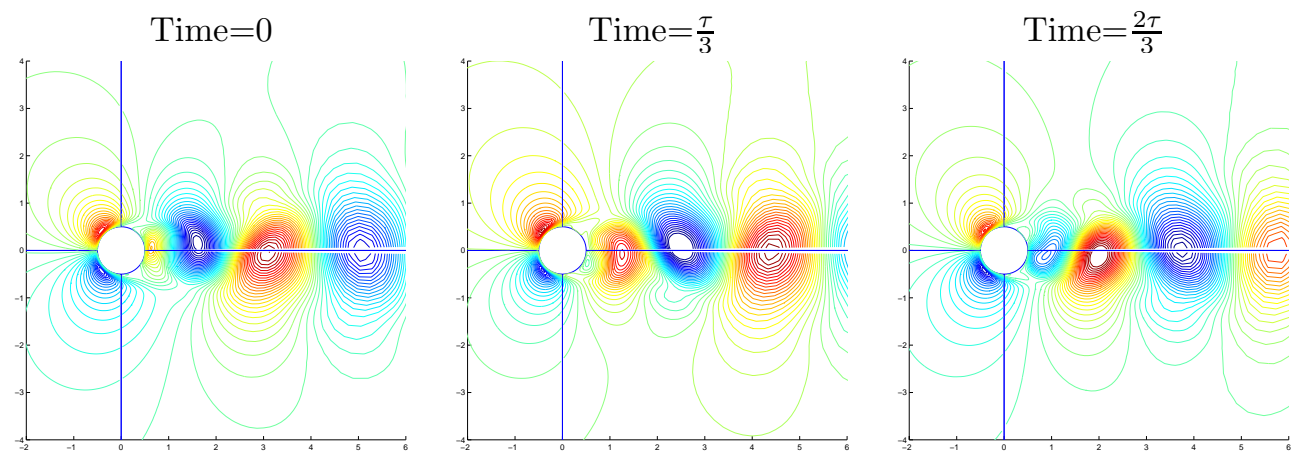

Fig. 11 Contours of the Y Component of Velocity - 1 Harmonic
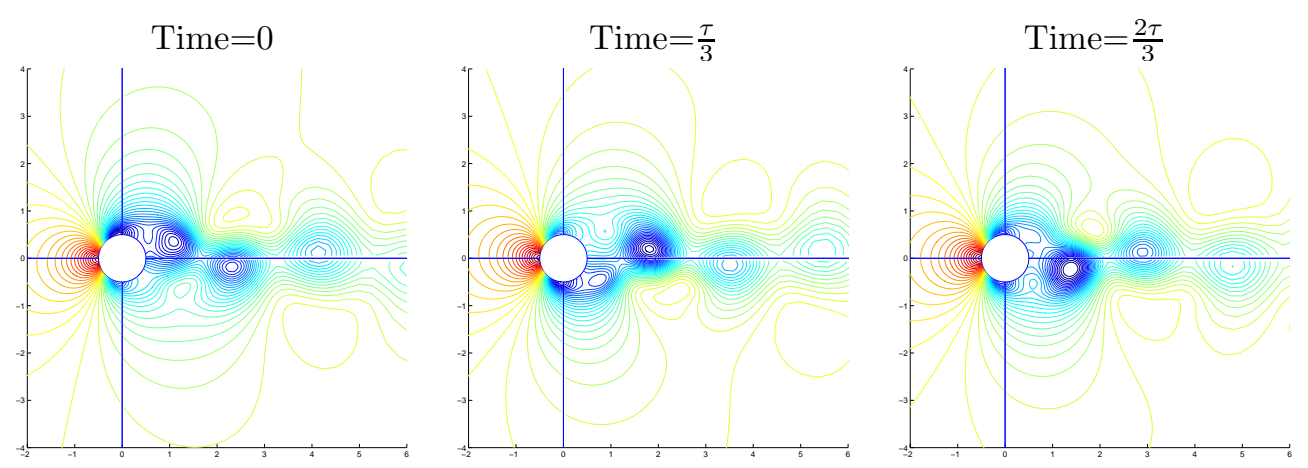

Fig. 12 Contours of Pressure - 1 Harmonic


Fig. 13 Contours of Entropy - 1 Harmonic

10 of 11 
Time $=0$
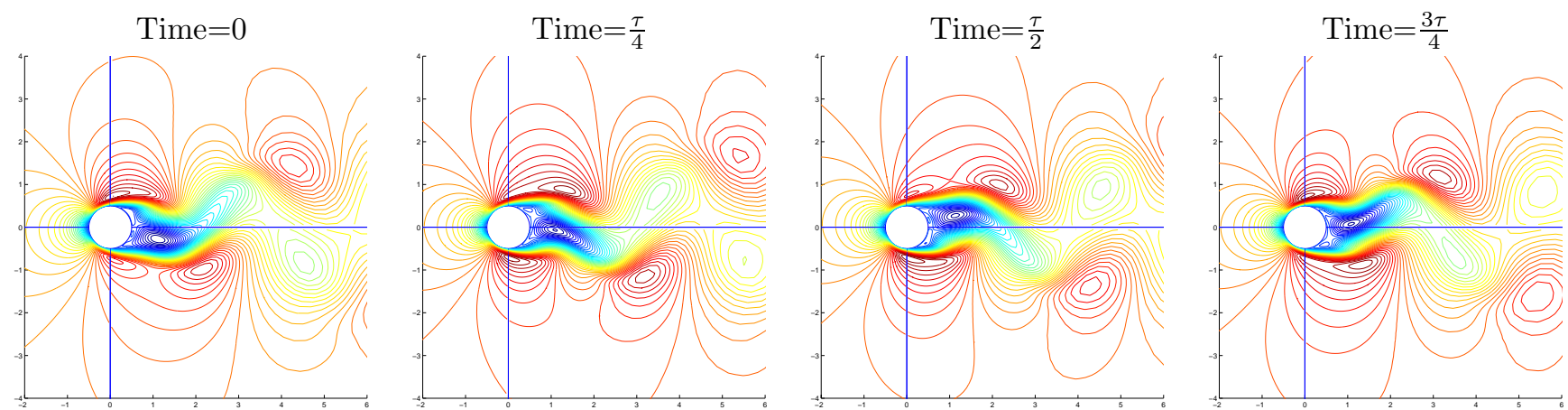

Fig. 14 Contours of the X Component of Velocity - 7 Harmonics

Time $=0$



Fig. 15


Contours of the Y Component of Velocity - 7 Harmonics
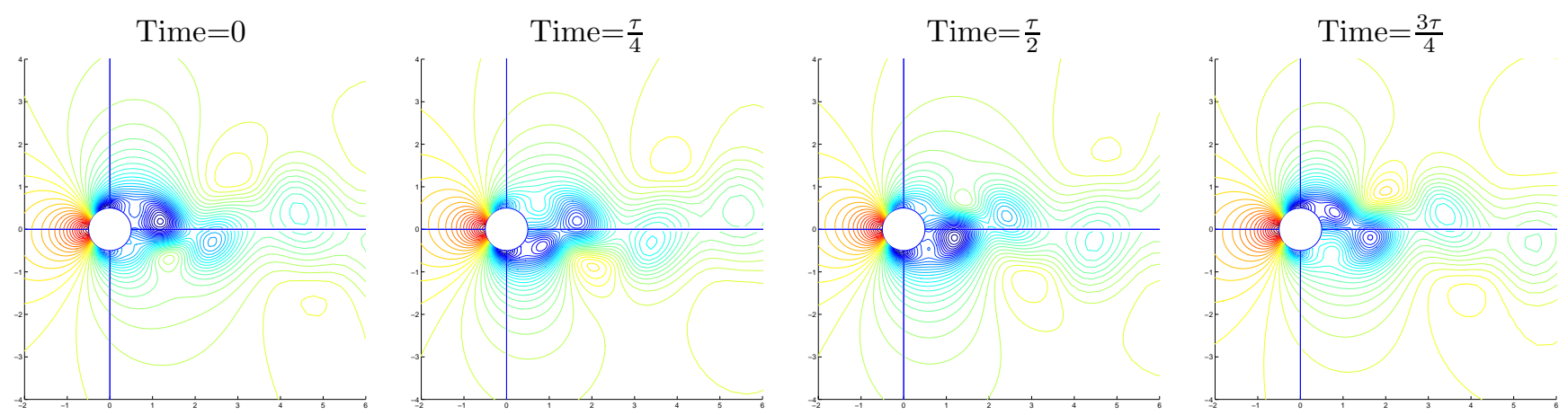

Fig. 16 Contours of Pressure - 7 Harmonics
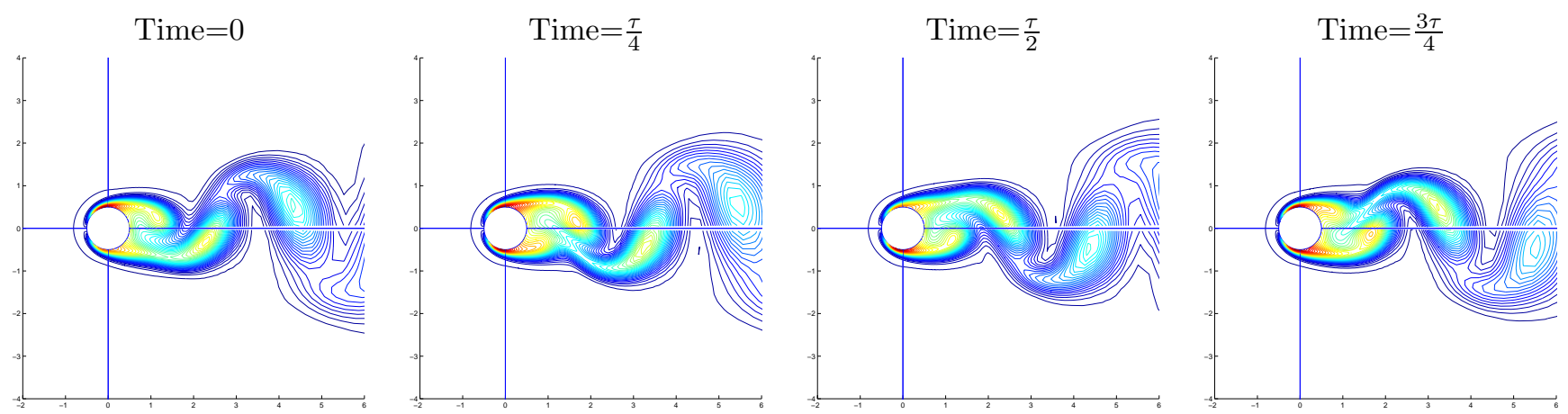

Fig. 17 Contours of Entropy - 7 Harmonics 